\title{
Long-term ultraviolet variability of Seyfert galaxies
}

\author{
N. SUKANYA ${ }^{1}{ }_{(\mathbb{D}}$, C. S. STALIN ${ }^{2}$, P. JOSEPH $^{2}$, S. RAKSHIT $^{2,3}$, D. PRAVEEN ${ }^{4}$ and \\ R. DAMLE ${ }^{1, *}$ \\ ${ }^{1}$ Department of Physics, Bangalore University, Bengaluru 560 056, India. \\ ${ }^{2}$ Indian Institute of Astrophysics, Block II, Koramangala, Bengaluru 560 034, India. \\ ${ }^{3}$ Department of Physics and Astronomy, Seoul National University, Seoul 08826, Republic of Korea. \\ ${ }^{4}$ Physics Department, Amrita School of Engineering, Bengaluru 560 035, India. \\ *Corresponding author. E-mail: ramkrishnadamle@ bub.ernet.in
}

MS received 29 September 2017; accepted 13 August 2018; published online 22 November 2018

\begin{abstract}
Flux variability is one of the defining characteristics of Seyfert galaxies, a class of active galactic nuclei (AGN). Although these variations are observed over a wide range of wavelengths, results on their flux variability characteristics in the ultraviolet (UV) band are very limited. We present here the long-term UV flux variability characteristics of a sample of fourteen Seyfert galaxies using data from the International Ultraviolet Explorer acquired between 1978 and 1995. We found that all the sources showed flux variations with no statistically significant differences in the amplitude of UV flux variation between shorter and longer wavelengths. Also, the flux variations between different near-UV (NUV, 1850-3300 $)$ and far-UV (FUV, 1150-2000 passbands in the rest frames of the objects are correlated with no time lag. The data show indications of (i) a mild negative correlation of UV variability with bolometric luminosity and (ii) weak positive correlation between UV variability and black hole mass. At FUV, about $50 \%$ of the sources show a strong correlation between spectral indices and flux variations with a hardening when brightening behaviour, while for the remaining sources the correlation is moderate. In NUV, the sources do show a harder spectrum when brighter, but the correlation is either weak or moderate.
\end{abstract}

Keywords. Active galaxies—Seyferts—variability.

\section{Introduction}

Active galactic nuclei (AGN) with observed bolometric luminosities of around $10^{11}-10^{14} \mathrm{~L}_{\odot}$, and that includes Seyfert galaxies amongst its class, are believed to be powered by accretion of matter onto supermassive black holes residing at the centres of galaxies (Lynden-Bell 1969; Rees 1984). According to the standard picture, accretion leads to the formation of an accretion disk that emits black body radiation. The observed ultraviolet (UV)/optical radiation in AGN is well represented by the superposition of several multi-temperature black body components (Frank et al. 2002) and the observed big blue bump (BBB) in AGN spectra is often attributed to the accretion disk.

AGN are known to show flux variations since their discovery and is now considered one of their defining characteristics (Wagner \& Witzel 1995; Ulrich et al. 1997). Such flux variations are seen on a range of time scales from a fraction of hours to years over the complete electromagnetic spectrum from low-energy radio to high energy $\gamma$-rays (Wagner \& Witzel 1995; Ulrich et al. 1997; Zhang \& Feng 2017; Giveon et al. 1999). In spite of having a wealth of monitoring data on large samples of AGN with varying time resolutions through time domain surveys as well as dedicated monitoring programs, the physical mechanisms that cause AGN flux variations are still not well understood. Although different physical processes contribute to the emission at different wavebands, the UV-optical emission is believed to be emitted from an optically thick and geometrically thin accretion disk (Frank et al. 2002). Therefore, study of flux variations in the UV/optical bands can enable one to understand the processes happening in the accretion disk of AGN in particular in the non-blazar category of AGN.

Earlier efforts on the study of UV variations in AGN were by Paltani and Courvoisier (1994), who carried out a systematic analysis of the flux variations in the 
UV of different classes of AGN using data from the international Ultraviolet Explorer (IUE) covering the period 1978-1991. Also, UV variability of blazars has been studied using IUE data (Edelson et al. 1991; Edelson 1992). According to their analysis, blazars show stronger variability at shorter wavelengths than at longer wavelengths. Subsequent to the work of Paltani and Courvoisier (1994), Welsh et al. (2011) carried out a systematic study of the UV variability of a large number of AGN using data from the Galaxy Evolution Explorer (GALEX) database. According to Welsh et al. (2011), the UV variability of quasars is much more than their optical fluctuations and among the UV bands, the variability observed in the far-UV (FUV; 1344-1786 band is larger than the variability in the near-UV (NUV; 1771-2831 $\AA$ ) band, which is also similar to that found by Paltani and Courvoisier (1994). The analysis of Paltani and Courvoisier (1994) failed to find any significant differences between the UV properties of radio-loud and radio-quiet quasars, prompting the authors to suggest that the UV emission from AGN is independent of the radio emission properties. Studies of optical flux variations in different categories of AGN indicate that blazars tend to show large amplitude and high duty cycles of variability within a night as compared to other radio-loud and radio-quiet AGN (Stalin et al. 2004). On year-like time scales, among Seyfert galaxies in the optical band, radio-loud sources are more variable than their radio-quiet counterparts (Rakshit \& Stalin 2017).

Short-time-scale UV flux variations of the order of 1000 to $10000 \mathrm{~s}$ were found in the Seyfert 1 galaxy NGC 7469 by Welsh et al. (1998), using the Faint Object Spectrograph on the Hubble Space Telescope as well as Fairall 9 (Lohfink et al. 2014). Most of the studies on the UV flux variability of AGN (Sakata et al. 2011; Paltani \& Courvoisier 1994) either using spectroscopy or broadband photometry indicate that the UV flux variability characteristics of AGN can be well described by accretion disk models. Vanden Berk et al. (2004), based on two epochs of observations on a large number of quasars, found a spectral hardening of the UV continuum emission with increasing flux values. Similar results were also found by Wilhite et al. (2005) using spectroscopic observations. Paltani and Walter (1996), using IUE observations, observed that the spectra of Seyfert galaxies vary with time and they become flatter when the source brightens. To explain the observations, they proposed a two component model wherein the UV flux variations consist of a variable component with a constant spectral shape and a non-variable component from the small blue bump (SBB). Also, there are reports that claim the constancy of UV spectral shape during flux variations of AGN (Alloin et al. 1995; RodriguezPascual et al. 1997). As we have limited studies on the UV flux variability characteristics of AGN both in the long term as well as short term, it is of great importance to expand the studies on the already known UV flux variability nature of AGN to a larger sample of sources, and having data for a longer duration of time than that analysed earlier by Paltani and Walter (1996). Towards this, we have carried out a statistical analysis of the UV variability of a sample of Seyfert galaxies, a category of AGN for which sufficient data is available and focussed mainly on the FUV (1150-2000 $)$ and NUV (1850-3200 ̊) flux variations.

\section{Sample and data}

Our sample of Seyfert 1 galaxies was taken from Dunn et al. (2006) who have provided continuum light curves in different wavebands for a sample of 175 Seyfert galaxies as part of the Program in Extra Galactic Astronomy (PEGA). ${ }^{1}$ The data in this compilation were taken from the observations carried by IUE between the period 1978 and 1995. In this database, Dunn et al. (2006) have provided continuum flux measurements at three line-free regions in the spectra of each of the Seyfert galaxies. In IUE spectra, the NUV and FUV cover the wavelength regions $1850-3200 \AA$ and 1150-2000 A respectively. For most of the sources, flux measurements are available in three NUV passbands (2200, 2400 and $2740 \AA$ ) with bin sizes of 50, 60 and $30 \AA$ and three FUV passbands (1355, 1720 and $1810 \AA$ ) with bin sizes of 30,30 and $50 \AA$. For this study, we have downloaded the light curves for all the Seyfert galaxies that are available in the PEGA database and we applied the following conditions to select the light curves for further analysis:

1. The sources must have data from the two cameras of IUE, namely, the short-wavelength prime (SWP) and long-wavelength prime (LWP).

2. The total number of points (that includes all the three continuum passbands in FUV and NUV) must be larger than 50 .

The above two conditions led us to a final sample of 14 Seyfert galaxies spanning the redshift range $0.002<z<0.07$. Of the 14 selected Seyfert galaxies, one galaxy (NGC 1068) belongs to the Seyfert 2 category (having narrow permitted and forbidden lines),

\footnotetext{
${ }^{1}$ http://www.astro.gsu.edu/PEGA/IUE.
} 
while the remaining 13 sources belong to the Seyfert 1 category with broad permitted lines and narrow forbidden lines. The details of the objects selected for this study are given in Table 1. In this table, the total in column 7 refers to the total number of photometric points for a source in all the six passbands together. The entries against $\lambda_{1}, \lambda_{2}$ and $\lambda_{3}$ in SWP and LWP columns refer to the central wavelength used for the photometry and $\mathrm{N}_{N U V}$ and $\mathrm{N}_{F U V}$ give the number of points in each of the NUV and FUV passbands.

The observed flux values in all the six passbands were corrected for galactic extinction using the $A_{V}$ values taken from NED, ${ }^{2}$ which uses Schlafly and Finkbeiner (2011) and the extinction law evaluated in the UV range using the formalism given by Cardelli et al. (1989). The galactic extinction corrected flux values were then subjected to further analysis. We note here that the measured flux values were not corrected for extinction due to the host galaxies of the sources. The light curves in all the FUV and NUV passbands for the sources are given in Fig. 1 through Fig. 5. In these figures, the quoted wavelengths are in the observed frame of the sources. The present sample analysed here has some overlap with that reported by Paltani and Walter (1996). The sample analysed by Paltani and Walter (1996) has 15 sources that includes Seyfert galaxies, radio-loud as well as radio-quiet quasars. Their analysis was based on IUE data collected up to 1991. Our sample analysed here contains 14 Seyfert galaxies using data from IUE upto 1995. Though there are 10 sources in common to the sample reported here and that of Paltani and Walter (1996), the data analysed here is more extended in terms of the number of epochs and the total duration (1978-1995) as compared to Paltani and Walter (1996), who have analysed data from IUE until 1991.

\subsection{Flux variability}

For all the 14 sources selected based on the criteria outlined above, we carried out analysis to characterise their variability. This was done by calculating their normalized excess variance defined in Vaughan et al. (2003) as

$F_{v a r}=\sqrt{\frac{S^{2}-\overline{\sigma_{e r r}^{2}}}{\bar{x}^{2}}}$

where $S^{2}$ and $\sigma_{\text {err }}^{2}$ are the sample variance and average error defined as

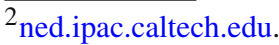

$$
\begin{aligned}
S^{2} & =\frac{1}{N-1} \sum_{i}\left(x_{i}-\bar{x}\right)^{2} \\
\overline{\sigma_{e r r}^{2}} & =\frac{1}{N} \sum_{i=1}^{N} \sigma_{e r r, i}^{2}
\end{aligned}
$$

The error in $F_{v a r}$ was calculated again using Vaughan et al. (2003) and is defined as

$\sigma_{F_{v a r}}=\sqrt{\left(\sqrt{\frac{1}{2 N} \frac{\sigma_{\text {err }}^{2}}{\bar{x}^{2} F_{v a r}}}\right)^{2}+\left(\sqrt{\frac{\overline{\sigma_{\text {err }}^{2}}}{N}} \frac{1}{\bar{x}}\right)^{2}}$

The majority of the sources in our sample have overlapping coverage in FUV and NUV passbands except for five sources, namely, 3C 120, 3C 390.3, NGC 3516, NGC 7469 and NGC 4051. This is evident in the light curves of these sources shown in Figs. 1, 3 and 5. Because of this, for calculating $\mathrm{F}_{v a r}$, we have considered only those duration of the light curves that have overlapping coverage in both FUV and NUV passbands. The calculated $\mathrm{F}_{\text {var }}$ values for all the sources in each of the six continuum passbands are given in Table 2 . A source is considered variable if its $F_{v a r}$ is greater than zero, and it is significant at the one sigma level. In all instances in our sample, $\mathrm{F}_{v a r}$ is many times greater than their associated errors except in four cases where it is less than three times their associated errors. Among these four too, in two cases $\mathrm{F}_{\text {var }}$ is more than two times their associated errors and in the remaining two cases it is between one and two sigma. We therefore argue that all the sources in the sample analysed here are highly variable in all the six passbands, except in four instances where the variability is less significant. The mean $\mathrm{F}_{\text {var }}$ values in all the six passbands for all the sources studied here is shown in Table 3 . There is an indication that the variations at the shorter wavelengths are larger than those at the longer wavelengths, but the larger error bars preclude us to draw any firm conclusion on the differences in variability between different wavelengths. Clubbing the $\mathrm{F}_{\text {var }}$ values in the three SWP passbands together as SWP and the three LWP passbands together as LWP, we obtained simple average values of $F_{v a r}$ as $0.392 \pm 0.196$ and $0.293 \pm 0.152$ for SWP and LWP respectively. The total number of $\mathrm{F}_{\text {var }}$ values are thus 42, in each of the SWP and LWP passbands. The distributions of $F_{v a r}$ for SWP and LWP and their cumulative distributions are given in Figs. 6 and 7 respectively. The average value of $\mathrm{F}_{\text {var }}$ is larger in SWP than in LWP, however, as the error bars are larger we carried out a two sample KS test. The null hypothesis that was tested was that the two independent $\mathrm{F}_{v a r}$ values pertaining to SWP and LWP were drawn from the 


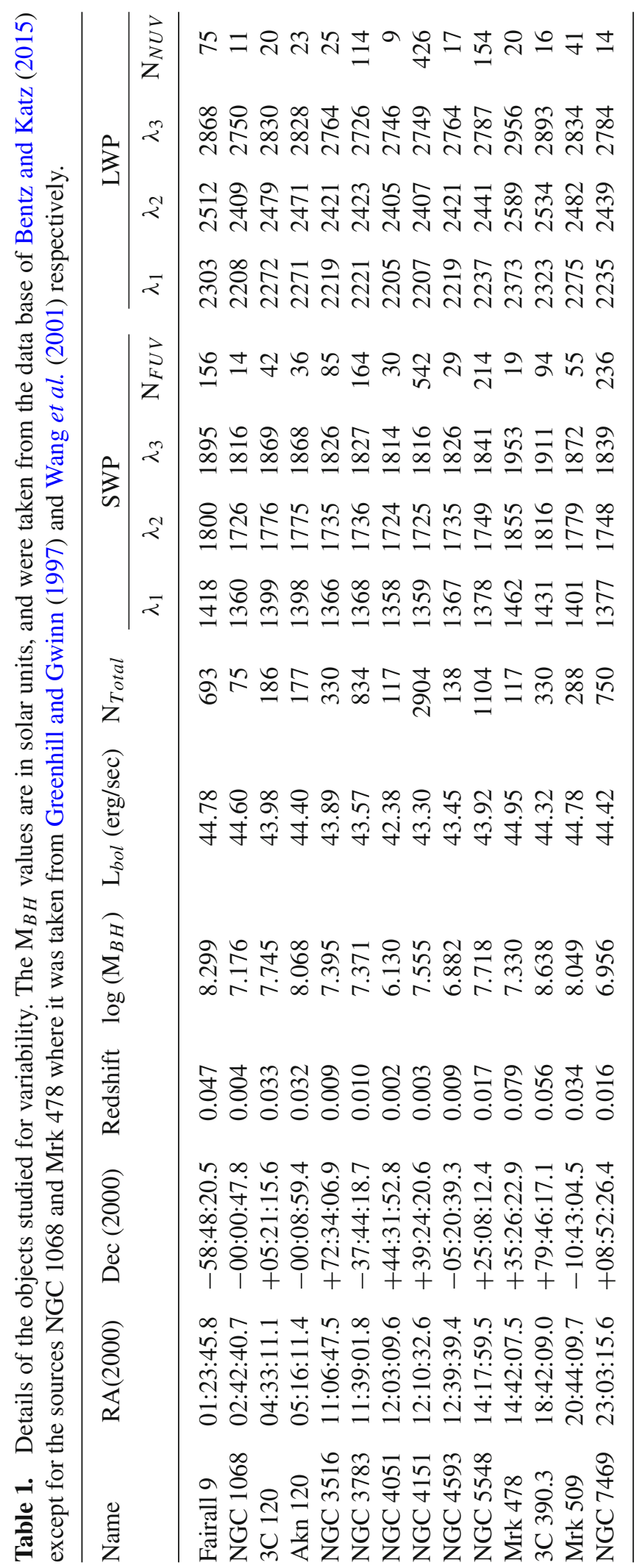



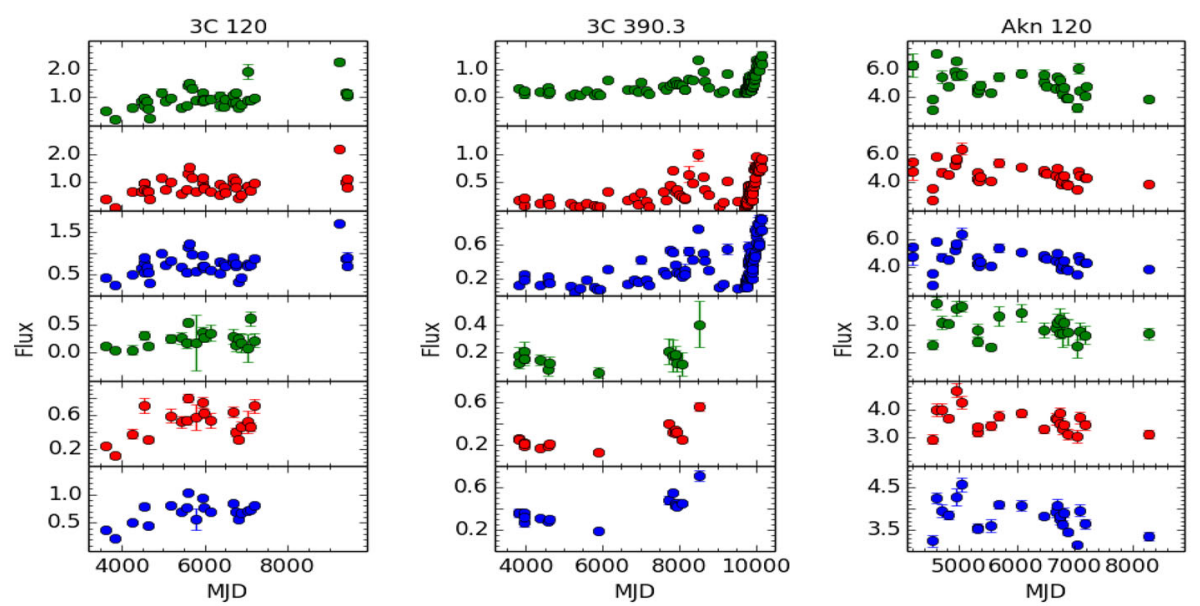

Figure 1. FUV and NUV light curves. The top three panels are for the FUV bands and the light curves in the bottom three panels are for NUV bands. For 3C 120, from top to bottom the wavelength of the light curves are $1399 \AA$, $1776 \AA$, $1869 \AA$, $2272 \AA, 2479 \AA$ and $2830 \AA$. For $3 \mathrm{C} 390.3$, the wavelength of the light curves from top to bottom are $1431 \AA, 1816 \AA, 1911 \AA$, $2323 \AA, 2534 \AA$ and $2893 \AA$. For Akn 120, the light curves shown from top to bottom are in wavelengths of $1390 \AA$, $1775 \AA$, $1868 \AA, 2271 \AA, 2477 \AA$ and $2828 \AA$.
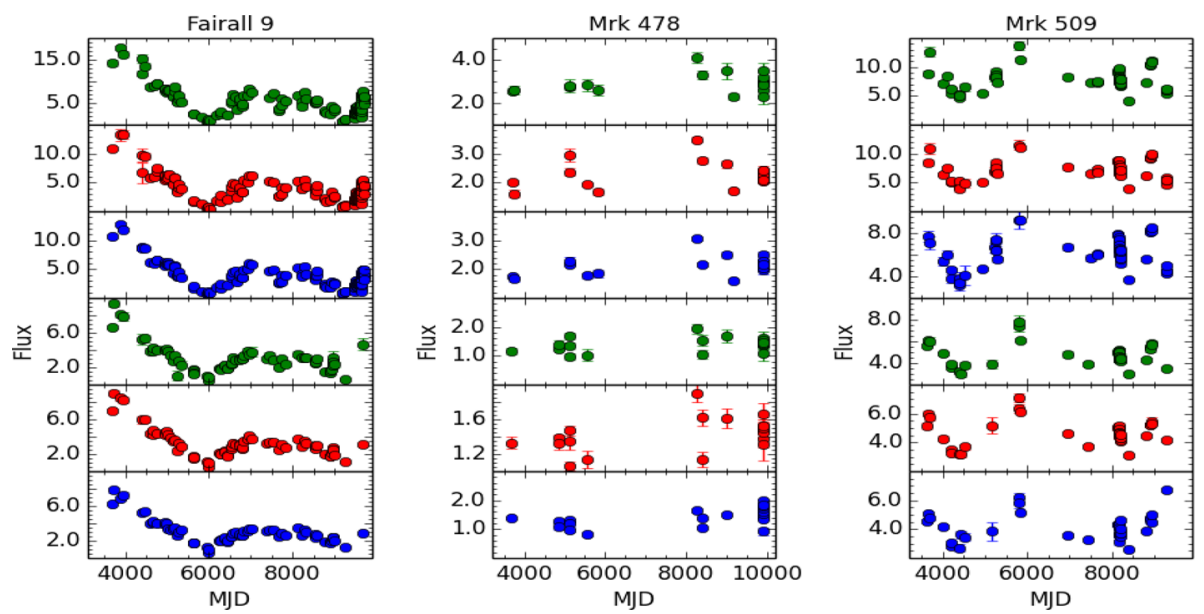

Figure 2. Light curves in increasing order of wavelengths from top to bottom for Fairall 9 (left), Mrk 478 (middle) and Mrk 509 (right). For Fairall 9, the wavelengths are $1418 \AA, 1800 \AA, 1895 \AA, 2303 \AA, 2512 \AA$ and 2868 AA. For Mrk 478 , the light curves from top to bottom have wavelengths of $1462 \AA, 1855 \AA, 1953 \AA, 2373 \AA, 2589 \AA$ and $2956 \AA$. For Mrk 509 , from the top to the bottom panels, the light curves have the wavelengths of $1401 \AA, 1779 \AA, 1872 \AA, 2275 \AA, 2482 \AA$ and $2834 \AA$.

same distribution. This null hypothesis was accepted, as $\mathrm{D}$ was lesser than the critical value of $\mathrm{D}\left(\mathrm{D}_{\text {crit }}\right)$. We obtained values of 0.286 and 0.356 for $\mathrm{D}_{\text {and }} \mathrm{D}_{\text {crit }}$ respectively for a significance level of 0.01 . This statistically points to no difference in the $\mathrm{F}_{v a r}$ values between SWP and LWP bands. Available studies do indicate that in UV, AGN show wavelength-dependent variability, with shorter wavelengths showing large amplitude of variability compared to the longer wavelengths (Sakata et al. 2011; Vanden Berk et al. 2004; Welsh et al. 2011). Data analysed here do indicate that variations at the shorter wavelengths are larger than that at longer wavelengths; however, due to the quality of the data, the error bars are too large to draw any conclusion on variation of amplitude of variability with wavelength.

\section{Correlation between variability and other physical properties}

\section{$3.1 F_{\text {var }}$ and $L_{b o l}$}

To find the presence of any correlation between $\mathrm{F}_{\text {var }}$ and bolometric luminosity $\left(\mathrm{L}_{b o l}\right)$, we plotted in Fig. 8 the variation of $\mathrm{F}_{v a r}$ with $\mathrm{L}_{b o l}$. The $\mathrm{F}_{v a r}$ values used in this correlation analysis is for the NUV band for the 

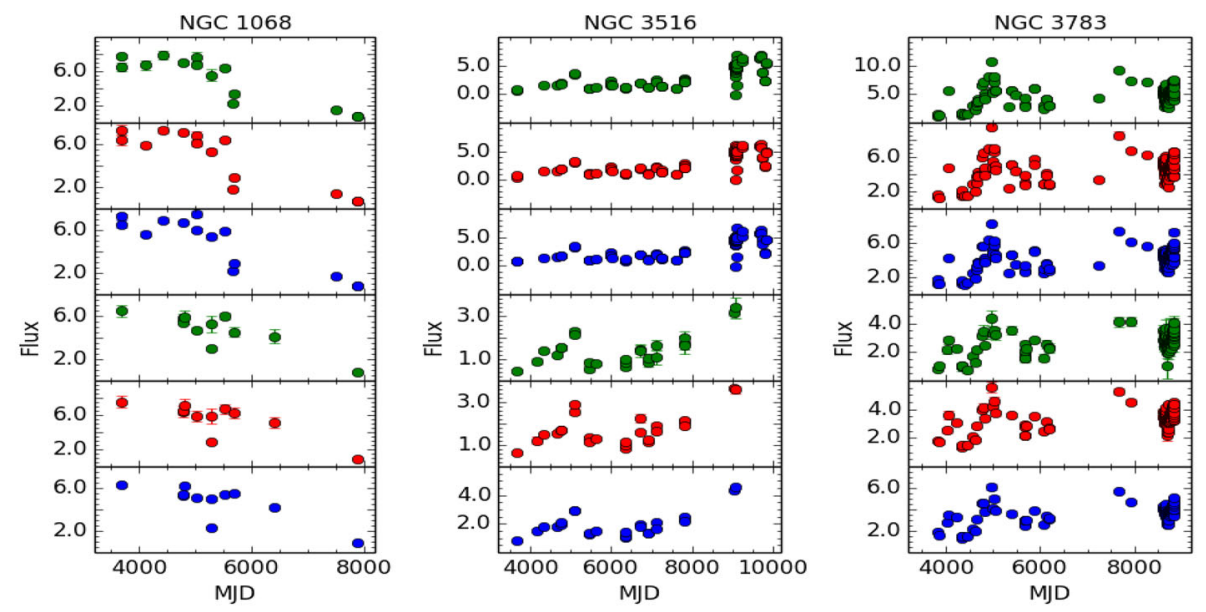

Figure 3. Light curves in FUV and NUV bands for the sources NGC 1068 (left), NGC 3516 (middle) and NGC 3783 (right). For NGC 1068 the light curves from the top to the bottom panels are at wavelengths of $1360 \AA$, $1726 \AA$, $1816 \AA$, $2208 \AA, 2409 \AA$ and $2750 \AA$. For NGC 3516 the light curves are at increasing order of wavelengths from top to bottom and have wavelength values of $1366 \AA$, $1735 \AA, 1826 \AA, 2219 \AA, 2421 \AA$ and $2764 \AA$. For NGC 3783 , the light curves from top to bottom panels have wavelengths of $1368 \AA, 1736 \AA, 1827 \AA, 2221 \AA, 2423 \AA$ and $2766 \AA$.
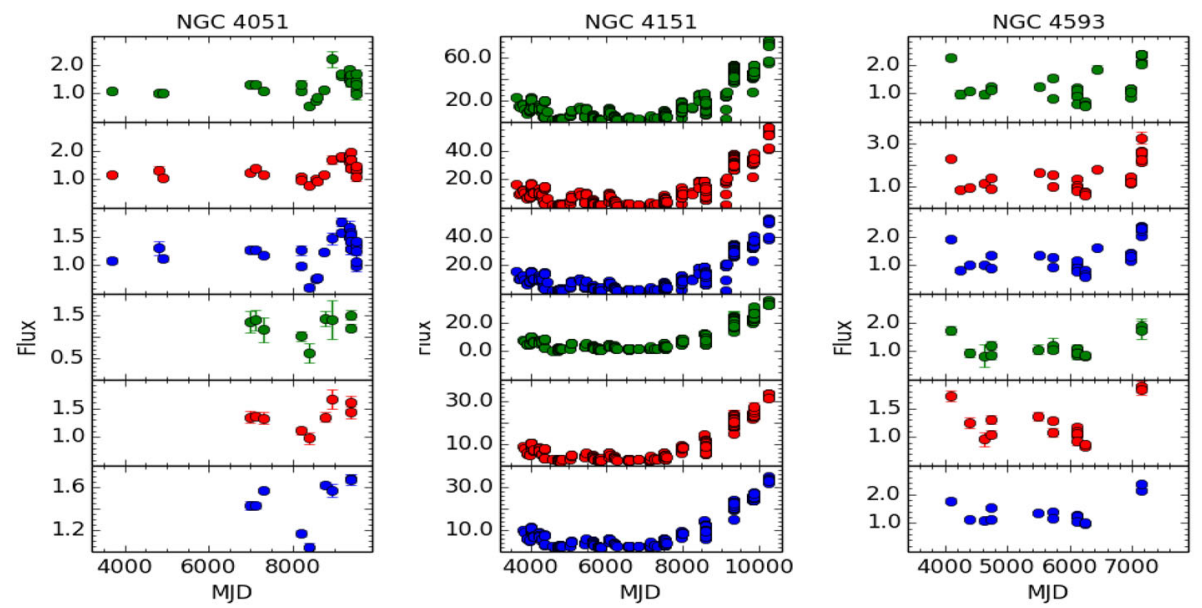

Figure 4. Light curves in FUV and NUV bands for the sources NGC 4051, NGC 4151 and NGC 4593. For NGC 4051, the light curves in increasing order of wavelength from top to bottom are at wavelengths of $1358 \AA, 1724 \AA, 1814 \AA, 2205 \AA$, $2405 \AA$ and $2746 \AA$. For NGC 4151, the light curves are at $1359 \AA, 1725 \AA, 1816 \AA, 2207 \AA, 2407 \AA$ and $2749 \AA$ from the top to the bottom panels. For NGC 4593, the shown light curves from top to bottom are for wavelengths of $1367 \AA, 1735 \AA$, $1826 \AA, 2219 \AA, 2421 \AA$ and $2764 \AA$.

passband $2806 \pm 64 \AA$. We used the relation $L_{b o l}=$ $13.2 \times L_{V}$ given by Elvis et al. (1994). Here, $L_{V}$ is the luminosity in the V-band which was derived using the V-band magnitude of the sources taken from SIM$\mathrm{BAD},{ }^{3}$ the zero-points taken from Bessel (1979) and the luminosity distance taken from NED. ${ }^{4}$ Using all the $\mathrm{F}_{\text {var }}$ values, we found indication of no correlation between $\mathrm{F}_{v a r}$ and $L_{b o l}$ with a low correlation coefficient of -0.08 and a probability of no correlation of

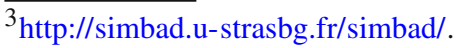

${ }^{4}$ http://www.astro.ucla.edu/ wright/CosmoCalc.html.
}

$P=0.79$. This trend for no correlation between $\mathrm{F}_{v a r}$ and $\mathrm{L}_{b o l}$ is due to one low luminosity source NGC 4051. Neglecting this source and doing a linear least squares fit to the data gave evidence for a mild negative correlation between $\mathrm{F}_{v a r}$ and $\mathrm{L}_{b o l}$. The linear least square fit is shown as a dashed line in Fig. 8. Correlation analysis indicates a mild negative correlation with a correlation coefficient of -0.39 with a probability of no correlation of $P=0.19$. This is in agreement with what is known in literature. Using IUE data, Paltani and Courvoisier (1997) found an anti-correlation between quasar variability and luminosity with high-luminosity 

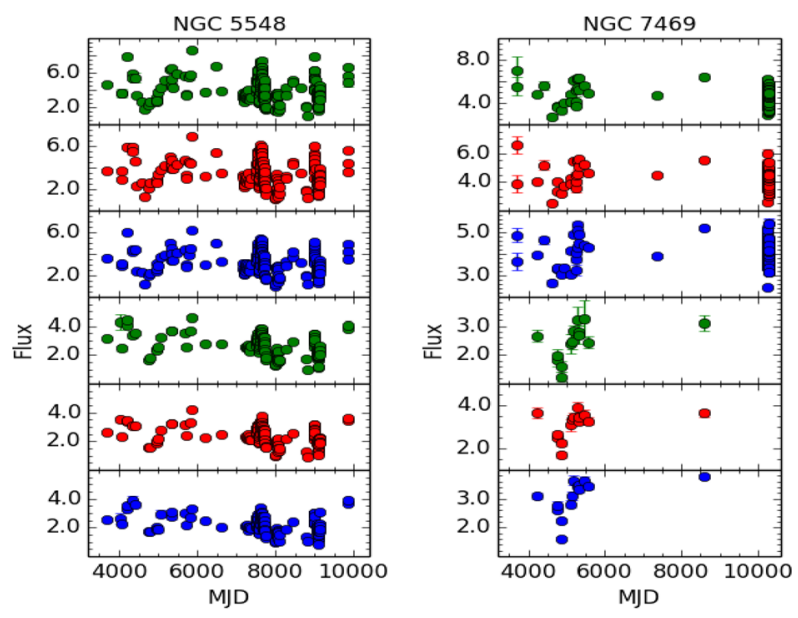

Figure 5. FUV and NUV light curves for the sources NGC 5548 and NGC 7469. For NGC 5548, the wavelengths of the light curves from top to bottom are $1378 \AA, 1749 \AA$, $1841 \AA, 2237 \AA, 2441 \AA$ and $2787 \AA$. For NGC 7469 the light curves shown from top to bottom have wavelengths of $1377 \AA$, $1748 \AA, 1839 \AA, 2235 \AA$ A, $2439 \AA$ and $2784 \AA$.

quasars showing low-amplitude of variability. This anticorrelation is also seen in the optical bands (Vanden Berk et al. 2004; Meusinge \& Weiss 2013). Analysing large sample of quasars for UV variability using data from GALEX, Welsh et al. (2011) found two different correlations between variability and luminosity. For time lags greater than 100 days, variability is negatively correlated with luminosity, while for time lags lesser than 100 days, variability is positively correlated with luminosity. The data analysed here too reveal a negative correlation between UV variability and luminosity. However, quality UV data (with similar time resolution and uniform coverage in both FUV and NUV) on a larger sample of sources are needed to firmly establish this finding.

\section{$3.2 F_{\text {var }}$ and $M_{B H}$}

Correlation between optical variability and black hole (BH) mass has been widely studied in the optical region with no clear consensus. From an analysis of the long term optical variability of quasars, Wold et al. (2007) found a correlation between variability and $\mathrm{BH}$ mass with sources with large BH mass showing larger amplitude of variability. Such a correlation was also noticed by Wilhite et al. (2008); however, Meusinge and Weiss (2013) and Zuo et al. (2012) could not find any correlation between optical variability and BH mass. From the data set analysed here, we looked for the existence of any correlation between UV variability and $\mathrm{BH}$ mass. In
Fig. 9 we show the correlation between $\mathrm{F}_{v a r}$ and $\mathrm{M}_{B H}$ where we found hint for a positive correlation between $\mathrm{F}_{v a r}$ and $\mathrm{M}_{B H}$. Correlation analysis gave a Pearson's rank correlation coefficient of 0.18 with probability for no correlation being 0.54 . The $\mathrm{F}_{\text {var }}$ values used in this correlation analysis too is in NUV for the passband $2806 \pm 64 \AA$.

\section{Spectral variability}

To know the spectral variability nature of the sources studied here, we examined the change in the spectral index relative to the flux of the sources. The optical to UV continuum slope of an AGN can be well represented as a power law, $F_{v} \propto v^{-\alpha}$, where $F_{v}$ is the observed flux density and $\alpha$ is the spectral index. For each of the sources studied here, we have observations in six UV passbands. We therefore calculated the spectral index by fitting a power law of the form

$F_{\lambda} \propto \lambda^{\alpha-2}$

For NUV, $\alpha$ was determined using the above power law fit to three measurements and for FUV again three measurements were used to derive $\alpha$. The variation of $\alpha$ thus deduced against the flux of the sources in both SWP and LWP are shown in Figs. 10 and 11 respectively. For SWP, we selected the shortest wavelength of three, and for LWP, we selected the shortest wavelength of the three observations. This was only for the purpose of defining the flux values. For the analysis of correlation between $\alpha$ and flux, we considered only those points where the error in $\alpha$ and flux values are lower than the associated values of $\alpha$ and fluxes. The data were fit with a straight line by (i) assigning equal weight to all the points and (ii) taking into account the errors in both $\alpha$ and flux values. The un-weighted linear least squares fits are shown by dashed lines in Figs. 10 and 11, while the weighted linear least squares fits are shown by solid lines. From weighted linear least squares fit to the data we find that, for most of the sources, their spectra do not show any significant changes during the flux variations; however, for few sources, we found clear evidence of a hardening of the spectra with increase in flux. For some sources, we see structures in the variation of $\alpha$ with flux. The spectrum is found to harden with increasing flux; however, limited to certain moderate flux values, beyond which the spectrum is nearly steady showing no change with flux. This is seen in the sources NGC 3783, NGC 4151, NGC 4593 and Fairall 9 in SWP. In LWP, this is evident in the sources NGC 4151 and Fairall 9. The results of the linear least squares fit to the variation 


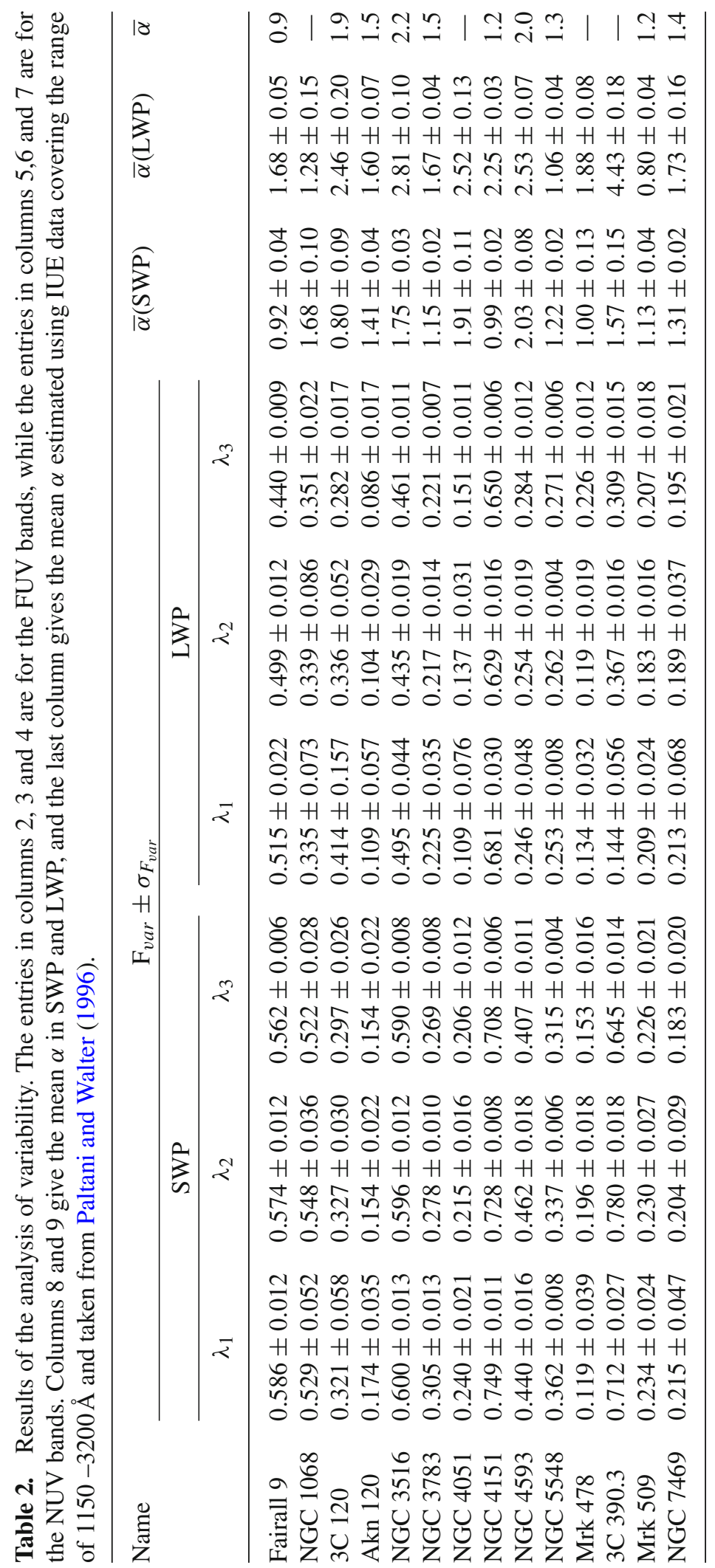


Table 3. Average $\mathrm{F}_{\text {var }}$ values for the different wavelength bands.

\begin{tabular}{cc}
\hline Mean wavelength & Mean $\mathrm{F}_{\text {var }}$ \\
\hline $1389 \pm 30$ & $0.399 \pm 0.198$ \\
$1762 \pm 38$ & $0.402 \pm 0.203$ \\
$1855 \pm 40$ & $0.374 \pm 0.188$ \\
$2255 \pm 49$ & $0.292 \pm 0.168$ \\
$2460 \pm 53$ & $0.291 \pm 0.148$ \\
$2806 \pm 64$ & $0.295 \pm 0.139$ \\
\hline
\end{tabular}

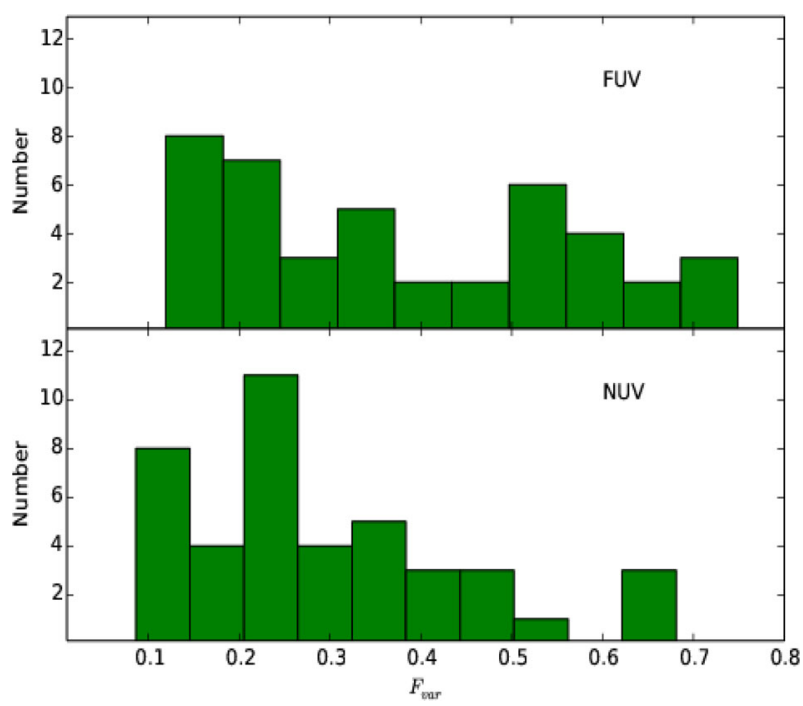

Figure 6. Distribution of $F_{v a r}$ values for the sources studied here in FUV (top panel) and NUV (bottom panel) bands.

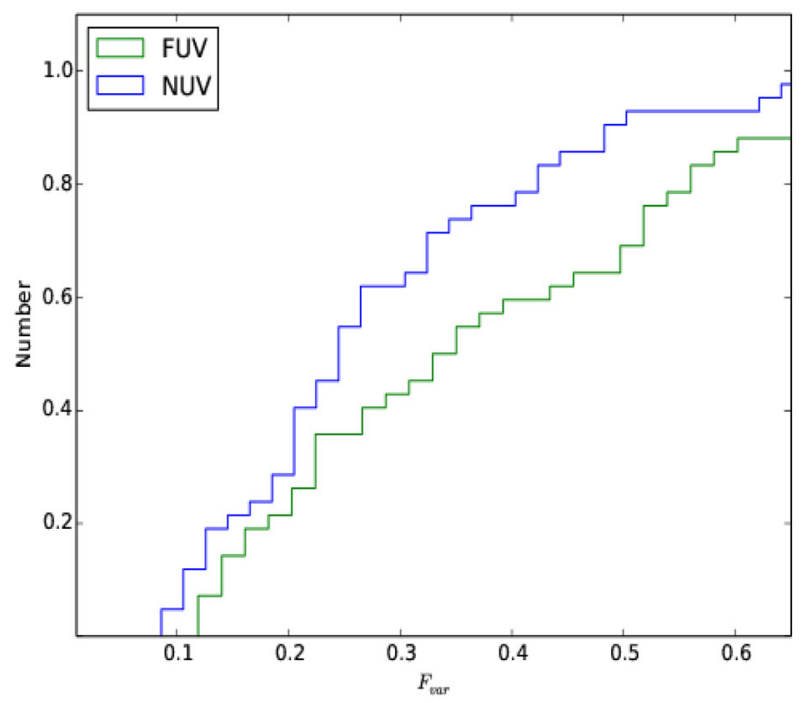

Figure 7. Cumulative distribution of the values of $\mathrm{F}_{\text {var }}$ in FUV (in green) and NUV (in blue) bands.

in $\alpha$ with flux is shown in Table 4 . In FUV about $50 \%$ of the sources showed a harder when brighter trend. The

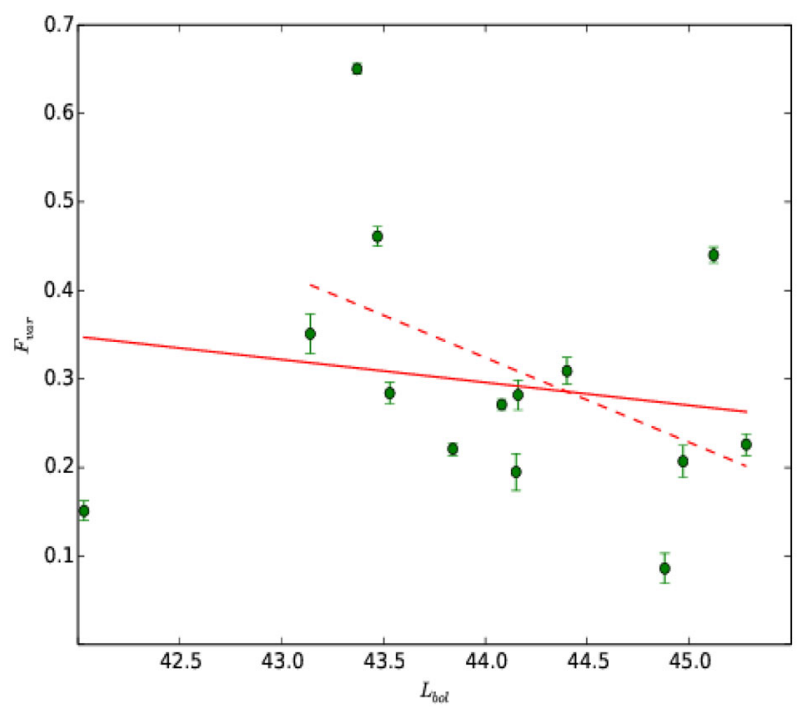

Figure 8. Variation of $\mathrm{F}_{v a r}$ with bolometric luminosity. Linear least squares fit to the data are shown for the complete data (solid line) and for the data set excluding the lowest luminosity source in our sample (dashed line).

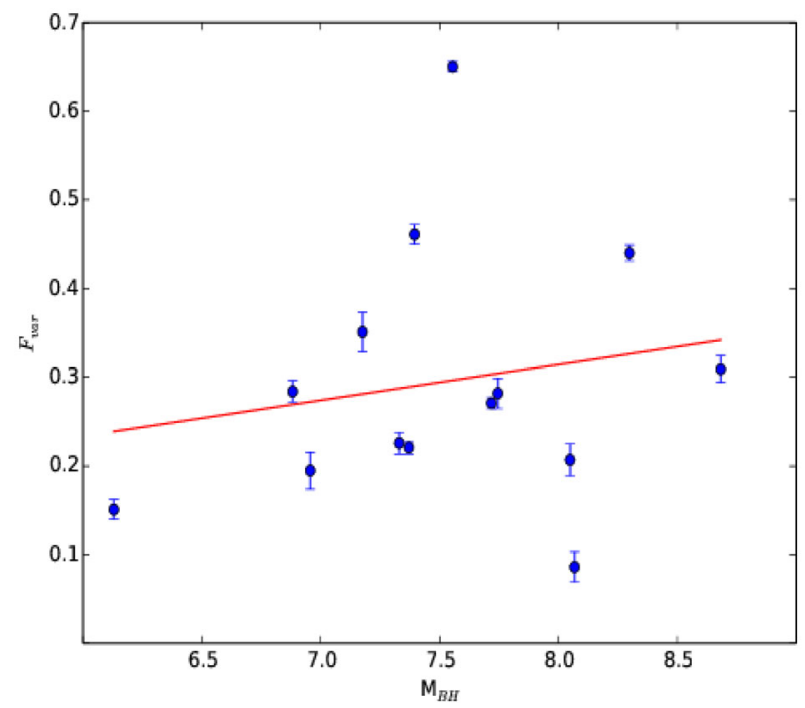

Figure 9. Plot of $\mathrm{F}_{\text {var }}$ against black hole mass. The solid line is the linear least squares fit to the data.

remaining sources too showed a harder when brighter behaviour but the correlation is moderate. The weighted and un-weighted linear least squares fit show similar trend for most of the sources, with the largest mismatch seen in Mrk 478. In the NUV band there is moderate correlation between $\alpha$ and the flux with a trend for a harder when brighter behaviour. Here too, large discrepancy between weighted and un-weighted linear least squares fits is seen in sources such as Mrk 478, NGC 4593 and 3C 390.3. These results to a large extent agree with the analysis of the UV continuum emission 

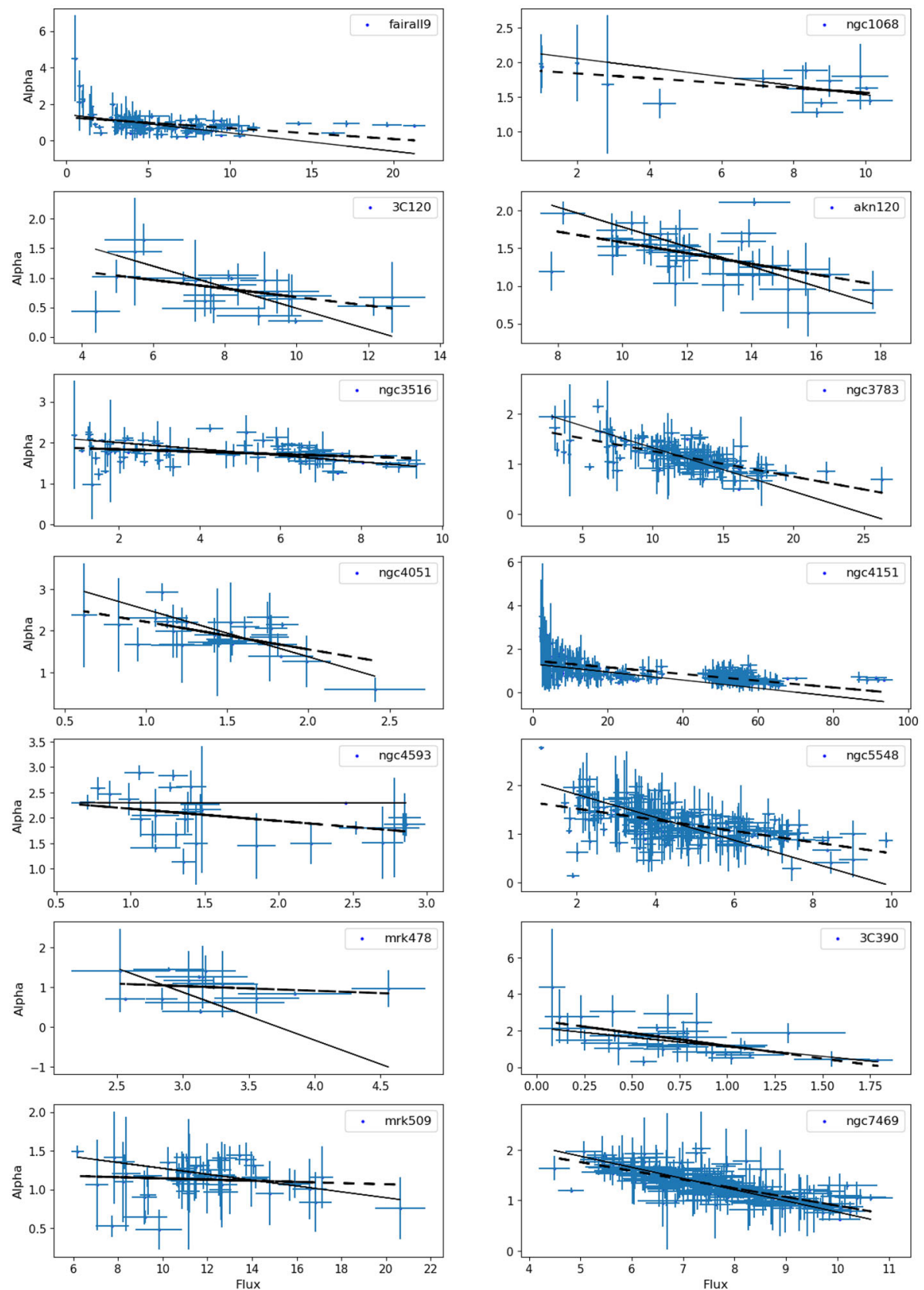

Figure 10. Variation of spectral index with flux in the shortest wavelength in FUV. The solid lines are the linear least squares fit to the data that take into account the errors in both $\alpha$ and fluxes, while the dashed lines are the un-weighted linear least squares fit to the data.

in AGN by Sakata et al. (2011), who also found a bluer when brighter trend in their sample. Similar conclusion was also arrived at by Wilhite et al. (2005) and Vanden Berk et al. (2004) in the optical band. Our results for a majority of the sources are also consistent with the observations of Paltani and Walter (1996) who found that the UV spectra of Seyfert galaxies becomes flatter with increased brightness of the sources. To explain these observations, Paltani and Walter (1996) proposed the two component model. According to this model, the observed flux is a superposition of two distinct spectral components, with constant spectral shapes. One component is flux variable while the other one is stable, and the observed continuum variation is driven by the amplitude of the varying component. For some sources in our sample such as NGC 3783, NGC 4151, NGC 4593 and Fairall 9, we in fact observed a constancy of the spectral index with increasing flux, however, only beyond 

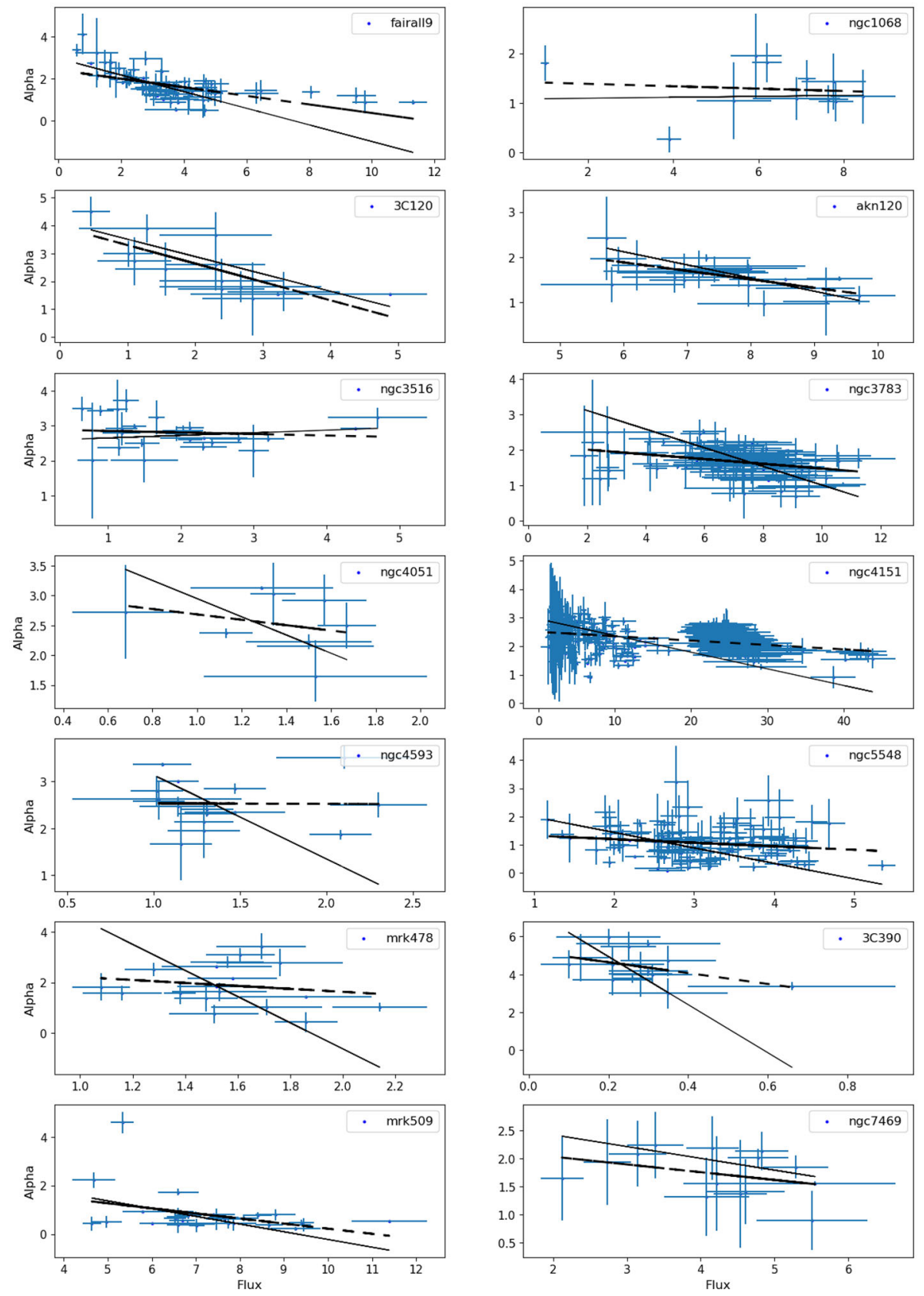

Figure 11. Variation of spectral index with flux in the NUV band. Linear least squares fit to the data that takes into account the errors in both the spectral indices and flux values are shown as solid lines. The un-weighted linear least squares fit to the data are shown with dashed lines.

certain flux levels in them. This points to the complex nature of UV flux variations in AGN (cf. Paltani \& Walter 1996). The mean values of $\alpha$ for the sources in SWP and LWP are given in Table 2. Also, given in the same table are the mean $\alpha$ values reported by Paltani and Walter (1996) estimated using IUE spectra covering the wavelength range of 1150-3200 A. For the sources that are in common between this study and that of Paltani and Walter (1996), the mean $\alpha$ values are similar, although the data analysed here is much more extensive than that of Paltani and Walter (1996).

\section{Lag between different wavebands}

To check for inter-band time lags we used the discrete correlation function (DCF) technique of Edelson and Krolik (1988). The cross-correlation analysis was done between the light curves of the shortest and longest 
Table 4. Results of the linear least squares fit between the variation of spectral indices and the fluxes at the shortest wavelength in NUV and FUV bands. The columns $r$ and $\mathrm{P}$ are the correlation coefficient and probability respectively.

\begin{tabular}{|c|c|c|c|c|c|c|c|c|}
\hline \multirow[b]{2}{*}{ Name } & \multicolumn{4}{|c|}{ SWP } & \multicolumn{4}{|c|}{ LWP } \\
\hline & Slope & Intercept & $r$ & $P$ & Slope & Intercept & $r$ & $P$ \\
\hline iirall 9 & $0.100 \pm 0.012$ & $1.425 \pm 0.083$ & 0.405 & 0.00 & $0.393 \pm 0.033$ & $2.965 \pm 0.105$ & 0.601 & 0.000 \\
\hline NGC 1068 & $-0.065 \pm 0.038$ & $2.188 \pm 0.353$ & 0.530 & & $0.009 \pm 0.078$ & $1.075 \pm 0.489$ & -0.109 & 0.751 \\
\hline 3C 120 & $-0.178 \pm 0.038$ & $2.268 \pm 0.326$ & 0.455 & 0.044 & $-0.622 \pm 0.116$ & $4.131 \pm 0.345$ & 0.760 & 0.002 \\
\hline Akn 120 & $-0.131 \pm 0.026$ & $3.097 \pm 0.301$ & 0.530 & 0.001 & $-0.291 \pm 0.071$ & $3.866 \pm 0.556$ & 0.653 & 0.001 \\
\hline NGC 3516 & $-0.079 \pm 0.007$ & $2.157 \pm 0.036$ & 0.286 & 0.008 & $0.074 \pm 0.026$ & $2.577 \pm 0.098$ & -0.101 & 0.637 \\
\hline GC 3783 & $-0.088 \pm 0.006$ & $2.204 \pm 0.068$ & 0.683 & 0.000 & $-0.262 \pm 0$. & $3.634 \pm 0.217$ & 0.329 & 0.000 \\
\hline GC 4051 & $-1.140 \pm$ & $3.653 \pm 0.326$ & 0.596 & 0.0 & $-1.525 \pm$ & $4.472 \pm 1.199$ & 0.285 & 0.457 \\
\hline GC 4 & $-0.019 \pm 0.001$ & $1.310 \pm$ & 0.720 & 0.0 & $-0.058 \pm$ & $2.951 \pm 0.090$ & 0.380 & 0.000 \\
\hline NGC 4593 & $-0.002 \pm 0.007$ & $2.302 \pm 0.018$ & 0.378 & 0.043 & $-1.798 \pm 0.626$ & $4.939 \pm 0.802$ & 0.010 & 0.971 \\
\hline NGC 5548 & $-0.234 \pm 0.019$ & $2.280 \pm 0.085$ & 0.547 & 0.000 & $-0.547 \pm 0.075$ & $2.533 \pm 0.250$ & 0.179 & 0.050 \\
\hline Mrk 478 & $-1.201 \pm 0.621$ & $4.474 \pm 1.896$ & 0.181 & 0.518 & $-5.171 \pm 2.293$ & $9.716 \pm 3.427$ & 0.177 & 0.483 \\
\hline 3C 390.3 & $-1.043 \pm 0.142$ & $2.160 \pm 0.172$ & 0.609 & 0.000 & $-12.695 \pm 4.974$ & $7.483 \pm 1.222$ & 0.445 & 0.084 \\
\hline Mrk 509 & $-0.038 \pm 0.017$ & $1.653 \pm 0.191$ & 0.097 & 0.485 & $-0.318 \pm 0.098$ & $2.956 \pm 0.722$ & 0.388 & 0.031 \\
\hline NGC 7469 & $-0.221 \pm 0.009$ & $2.982 \pm 0.067$ & 0.786 & 0.000 & $-0.211 \pm 0.093$ & $2.846 \pm 0.460$ & 0.360 & 0.206 \\
\hline
\end{tabular}

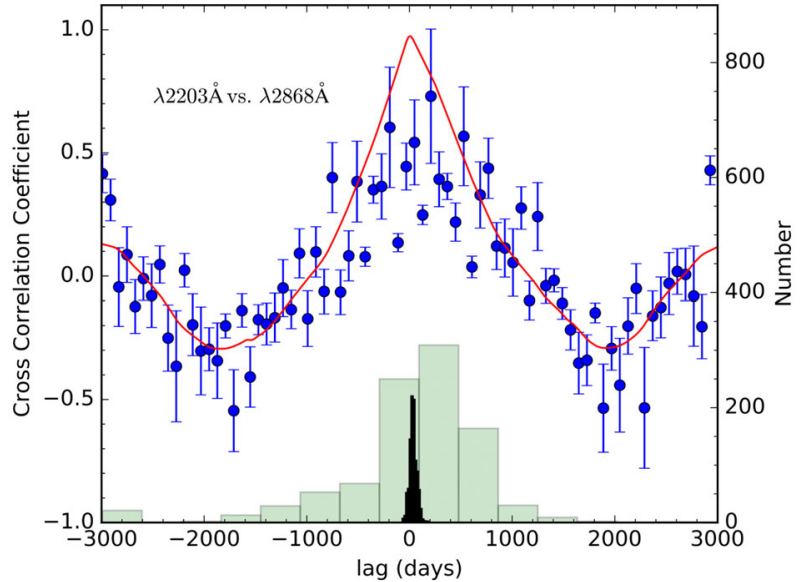

Figure 12. Cross-correlation analysis between the light curves at $2203 \AA$ and $2868 \AA$ for the source Fairall 9, one of the sources in the sample. The red solid line is for ICCF and the blue filled circles are for the DCF. The green and black histograms show the distribution of centroids for DCF and ICCF respectively.

wavelengths in both FUV and NUV for all the sources. We show in Fig. 12 the results on one correlation analysis for the object Fairall 9 carried out between the light curves at 2203 and $2868 \AA$. Here, the filled circles are those evaluated using the DCF method and the solid line is that obtained using the interpolated cross correlation function (ICCF) described in detail in Gaskell and Sparke (1986) and Gaskell and Peterson (1987). To evaluate the uncertainty in the derived lag, we followed a model of independent Monte Carlo approach that incorporated both flux randomization (FR) and random subset sampling (RSS) described in Peterson et al. (1998). For each Monte Carlo iteration, we found the lag using the centroid of the CCF utilizing all points within $60 \%$ of the peak of the CCF in the case of DCF. However, for ICCF the peak of the CCF was considered as a representation of the lag between the light curves. This was repeated for 10,000 times and the distribution of the CCF lags were obtained for both DCF and ICCF methods. The mean of the distributions were taken to represent the lag between the light curves and the spread in the distributions was used to estimate the error in the lag. The distributions obtained using both DCF (green histogram) and ICCF (black histogram) are given in Fig. 12 for the source Fairall 9. We found no noticeable time lag between flux variations in NUV and FUV bands, although the flux variations between different NUV and FUV bands were correlated. This analysis repeated for all the sources studied here yielded no measurable lags in any of them.

\section{Conclusion}

In the present work, we report the variability of fourteen Seyfert galaxies in the UV band using data from IUE acquired over a period of about 17 years. The flux values for the sources studied here in different NUV and FUV bands were taken from Dunn et al. (2006). 
Various analysis were performed to characterise the flux variability of the sources. The summary of the work is given below:

1. All sources were found to show flux variations in the UV band. No statistically significant difference in the amplitude of flux variations between shorter and longer wavelengths was noticed.

2. No time lag between flux variations in different NUV and FUV bands was observed.

3. We found a mild negative correlation of variability with bolometric luminosity with high luminous sources showing low variability than their less luminous counterparts. Also, a hint for a positive correlation is found between variability and black hole mass. These results are consistent with what is known in literature.

4. Majority of source showed a bluer when brighter trend in the FUV data; however, such a trend, if any in NUV band is seen only in a minority of the sources that too moderately. Some sources showed a hardening of the spectrum with flux, but the spectrum remained non-variable beyond certain flux level. The observed spectral variations are thus complex.

\section{Acknowledgements}

We thank the anonymous referee for his/her critical comments that helped to improve the manuscript

\section{References}

Alloin D. et al. 1995, A\&A, 293, 293A

Bentz M. C., Katz S. 2015, PASP, 127, 67

Bessel M. S. 1979, PASP, 91, 589

Cardelli J. A., Clayton G. C., Mathis J. S. 1989, ApJ, 345, 245

Dunn J. P., Jackson B., Deo R. P. et al. 2006, PASP, 118, 572

Edelson R. 1992, ApJ, 401, 516

Edelson R. A., Saken J., Pike G. et al. 1991, ApJL, 372, L9

Edelson R. A., Krolik J. H. 1988, ApJ, 333, 646

Elvis M. et al. 1994, APJ Supplement Series, 95, 1
Frank J., King A., Raine, D. J. 2002, in by Frank J., King A., Raine D., eds, Accretion Power in Astrophysics. Cambridge University Press, Cambridge, p. 398. ISBN 0521620538, February 2002

Gaskell C. M., Peterson B. M. 1987, ApJS, 65, 1

Gaskell C. M., Sparke L. S. 1986, ApJ, 305, 175

Giveon U., Maoz D., Kapsi S., Netzer H., Smith P. S. 1999, MNRAS, 306, 637

Greenhill L. J., Gwinn C. R. 1997, Ap\&SS, 248, 261

Lohfink A. M., Reynolds C. S., Vasudevan R., Mushotzky R. F., Miller N. A. 2014, Apj, 788, 10

Lynden-Bell D. 1969, Nature, 223, 690

Meusinge H., Weiss V. 2013, A\&A, 560, A104

Paltani S., Courvoisier T. J.-L. 1994, A\&A, 291, 74

Paltani S., Walter R. 1996, A\&A, 312, 55

Paltani S., Courvoisier T. 1997, A\&A, 323, 717

Peterson B. M., Wanders I., Horne K., Collier S., Alexander T., Kaspi S., Maoz D. 1998. The Publication of the Astronomical Society of the Pacific, 110(748), 660

Rakshit S., Stalin C. S. 2017, ApJ, 842, 96

Rees M. J. 1984, ARAA, 22, 471

Rodriguez-Pascual et al., A\&A, 72, 327

Sakata Y., Morokuma T., Minezaki T. et al. 2011, ApJ, 731, 50

Schlafly E. F., Finkbeiner D. P. 2011, ApJ, 737, 103

Stalin C. S., Gopal Krishna, Sagar R., Wiita P. J. 2004, Journal of Astrophysics and Astronomy, 25, 1

Ulrich M.-H., Maraschi L., Urry C. M. 1997, ARAA, 35, 445

Vanden Berk D. E., Wilhite B. C., Kron R. G., Anderson S. F., Brunner R. J., Hall P. B., Ivezić Ž., Richards G. T., Schneider D. P., York D. G. 2004, ApJ, 601, 692

Vaughan S., Edelson R., Warwick R. S., Uttley P. 2003, MNRAS, 345, 1271

Wagner S. J., Witzel A. 1995, ARAA, 33, 163

Wang X. Y., Dai Z. G., Lu, T. 2001 ApJ, 556, 1010

Welsh B. Y., Wheatley J. M., Neil J. D. 2011, A\&A, 527, A15

Welsh W. F., Peterson B. M., Koratkar A. P., Korista K. T. 1998, ApJ, 509, 118

Wilhite B. C., Vanden Berk D. E., Kron R. G., Schneider D. P., Pereyra N., Brunner R. J., Richards G. T., Brinkmann J. V. 2005, ApJ, 633, 638

Wilhite B. C., Brunner R. J., Grier C. J., Schneider D. P., Vanden Berk D. E. 2008, MNRAS, 383, 1232

Wold M., Brotherton M. S., Shang Z. 2007, MNRAS, 375, 989

Zhang X.-G., Feng L. 2017, MNRAS, 464, 2203

Zuo W., Wu X.-B., Liu Y.-Q., Jiao C.-L. 2012, ApJ, 758, 104 